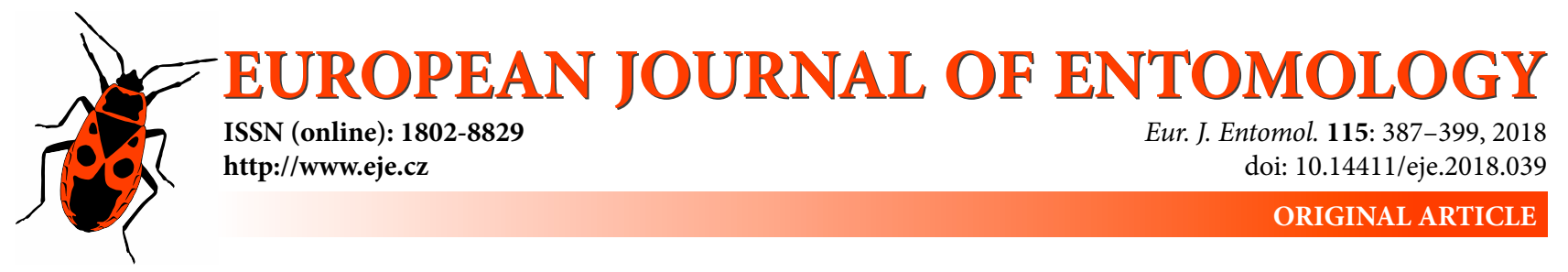

\title{
Discovery of a remarkable new species of Lymanopoda (Lepidoptera: Nymphalidae: Satyrinae) and considerations of its phylogenetic position: An integrative taxonomic approach
}

\author{
Tomasz W. PYRCZ ${ }^{1,5}$, Carlos PRIETO ${ }^{2,3}$, Pierre BOYER ${ }^{4}$ and JadWiga LORENC-BRUdeCKA ${ }^{5, *}$ \\ ${ }^{1}$ Entomology Department, Institute of Zoology and Biomedical Research, Jagiellonian University, ul. Gronostajowa 9, \\ 30-387 Kraków, Poland; e-mail: tomasz.pyrcz@uj.edu.pl \\ ${ }^{2}$ Corporación Universitaria Autónoma del Cauca, Calle 5 \# 3-87, Popayán, Colombia; e-mail: cprieto50@gmail.com \\ ${ }^{3}$ SNSB-Bavarian State Collection of Zoology, Münchhausenstr. 21, 81247 Munich, Germany \\ ${ }^{4}$ 7, Lotissement l'Horizon, 13610 Le Puy Sainte Réparade, France; e-mail: pierdom@aliceadsl.fr \\ ${ }^{5}$ Nature Education Centre, Jagiellonian University, ul. Gronostajowa 5, 30-387 Kraków, Poland; \\ e-mail: j.lorenc-brudecka@uj.edu.pl
}

Key words. Lepidoptera, Nymphalidae, Satyrinae, Lymanopoda, systematics, new species, phylogeny, DNA barcodes, biodiversity, páramo, Colombia, Andes, Valle del Cauca

\begin{abstract}
A new species of Lymanopoda Westwood, a cloud forest Neotropical genus of Satyrinae, is described from the páramo grasslands on an isolated, peripheral massif in the Colombian Central Cordillera of the Andes: L. flammigera Pyrcz, Prieto \& Boyer, sp. n. The genus Lymanopoda is species-rich (approx. 65 species) and its alpha taxonomy is relatively well researched. Relationships within the genus using molecular data have also been explored. The new species is outstanding for its golden yellow colour in males, not found in any other neotropical Satyrinae. Cladograms were constructed based on COI sequences of 47 species of Lymanopoda ( $70 \%$ of the known species) including 17 from Colombia. The new species segregates in the "tolima" clade, which comprises four other high altitude Colombian species, as well as two from Ecuador. However, it is the comparative analysis of male genitalia, in particular the superuncus and valvae, which identified its closest relatives, thus confirming that genital characters can help refine molecular phylogenies. In addition to identifying species using mitochondrial DNA (mtDNA barcodes), nucleotide sites with unique fixed states used to identify nine species of Lymanopoda from Colombia are also presented.
\end{abstract}

ZooBank Article LSID: F820B047-2E29-4DEC-9C23-BB9A5B076528

\section{INTRODUCTION}

Colour patterns of butterfly wings are among the most outstanding expressions of evolution. Under certain conditions they are extremely plastic and even dramatic changes can be controlled by simple genetic mechanisms and quickly respond to selective pressure, for example in Heliconius (Kronforst \& Papa, 2015). Colours have many adaptive roles, commonly in intraspecific sexual communication, warning, mimetic relations or, very frequently, crypsis. In different groups of butterflies different roles dominate. In the cosmopolitan subfamily Satyrinae, the prevailing adaptation is camouflage, and its over 2500 species are overwhelmingly dark with shadows of brown, thus their common English name (browns), with some elements enhancing their cryptic colouration, such as stripes and patches imitating the substrate, in most cases on the undersides of their wings. In a few genera, such as Elymnias Hübner, 1818 and Elymniopsis Fruhstorfer, 1907, showy colours are, however, dominant, which is explained by their involvement in Batesian mimicry rings (Mallet \& Joron, 1999). Among the few Satyrinae genera with species bearing conspicuous wing patches is the neotropical montane Lymanopoda Westwood. This genus can be considered as one of the best known among South American Satyrinae and there are a number of papers published, especially in the last two decades, on their taxonomy and distribution (Pyrcz, 1999, 2003, 2004, 2005, 2012; Pyrcz \& Boyer, 2011; Pyrcz \& Rodríguez, 2006; Pyrcz et al., 1999, 2009a, b, 2010, 2016), phylogenetics (Casner \& Pyrcz, 2010; Marín et al., 2016), ecology (Pyrcz \& Wojtusiak, 2002; Pyrcz \& Garlacz, 2012) and biology (Montero \& Ortíz, 2012). Many of the more than 60 species of

\footnotetext{
* Corresponding author; e-mail: j.lorenc-brudecka@uj.edu.pl
} 
Lymanopoda have conspicuous white, blue, and reddish ground colours, which may be marked with white or green patches. The underlying evolutionary rationale for this is still unknown but there is growing evidence that some kind of mimicry is involved (Pyrcz, in prep.). Yet, even in Lymanopoda the discovery of a species with shiny golden yellow males was extremely surprising as this kind of colouration is not only unique for the genus but also among all worldwide Satyrinae. Here we investigate its affinities within the genus Lymanopoda and address some questions about the adaptive role of its colour pattern.

\section{MATERIAL AND METHODS}

\section{Morphological studies}

Most of the material used in this study was obtained during field-work by C. Prieto and P. Boyer in Colombia. Specimens used for morphological studies were examined in the Nature Education Centre (formerly Zoological Museum) of the Jagiellonian University in Kraków (CEP-MZUJ). Types and additional specimens were examined in major public museums including Instituto de Ciencias Naturales de la Universidad Nacional, Bogotá, Colombia (ICN), the Natural History Museum, London, UK (NHMUK), Museo de Agronomía de la Universidad Central, Maracay, Venezuela (MIZA), Staatliches Museum für Tierkunde, Dresden, Germany (MTD) and Zoologische Museum, Humboldt Universität, Berlin, Germany (ZMHB), as well as in the collections of Pierre Boyer (PB) and Carlos Prieto (RCCP).

The terminal parts of the abdomens (including the genitalia) were removed from the specimens and soaked in $10 \% \mathrm{KOH}$ solution for 5-10 min. Subsequently, abdomens were preliminarily cleaned using soft tissue in water in order to expose genital parts. Water was removed from dissected genitalia using $90 \%$ and $95 \%$ solutions of ethanol. A Nikon digital camera DS-Fi1 and an Olympus SZX9 stereomicroscope were used for taking pictures of the dissections, which were then processed in Adobe PhotoShop 7.0 CE and Corel PHOTO-PAINT X3 programs to enhance focus and improve quality. The dissected genitalia were kept in glycerol in vials pinned under the corresponding specimens. Genital terminology largely follows Klots (1956). Adults were photographed using a Minolta E-500 digital camera. Colour plates were composed using Adobe PhotoShop version 8. The following abbreviations are used in the text: FW - forewing; HW - hindwing; D - dorsum; V - venter; HDP - hindwing dorsal median patch.

\section{Material and sampling area}

Partial nucleotide sequences of mtDNA cytochrome c oxidase subunit I gene (COI) of individuals from several populations occurring in the Andes in Colombia that were previously identified morphologically, were analyzed. Tissue samples were extracted from identified pinned specimens collected in the past 10 years, as it is less likely that sequence data can be obtained from old material. Altogether 79 specimens, representing 47 species, yielded a DNA sequence of over 400 base pairs (bp) in length. Specimens with shorter sequences were excluded from the analyses.

\section{Molecular delimitation of species and barcodes}

For the DNA analyses, 79 individuals of 47 species of Lymanopoda were included as well as 2 individuals of two different genera as an outgroup, Corades chelonis Hewitson and Lasiophila zapatoza (Westwood). One or two legs were removed from each dried specimen and stored in individual tubes. DNA extraction, amplification and sequencing of the barcode region of the COI gene were carried out at the Canadian Centre for DNA Barcod- ing (CCDB), Ontario, Canada, using standard high throughput protocols (Ivanova et al., 2006; deWaard et al., 2008). PCR amplification with a single pair of primers consistently recovered a 658 bp region near the 5 ' end of COI that included the standard 648 bp barcode region for the animal kingdom (Hebert et al., 2004). Complete specimen data including images, voucher deposition, GenBank accession numbers, GPS coordinates, sequences and trace files are accessible in the Barcode of Life Data System (BOLD) (Table 3).

Sequence divergences for the barcode region were quantified using the Kimura 2 Parameter model, employing the analytical tools in BOLD (BOLD alignment, pairwise deletion). This was done to determine whether there is a barcode gap (a break in the distribution among genetic distances of specimens belonging to the same species and those of specimens from different species), that would allow the identification of the specimens examined. Genetic distances between species are reported as minimum pairwise distances, while intraspecific variation is reported as mean and maximum pairwise distances.

Several quantitative species delimitation algorithms for molecular data have been developed over the past decade, including approaches dedicated to DNA barcodes such as Automatic Barcode Gap Discovery (ABGD) and Refined Single Linkage (RESL) Analysis algorithm (Puillandre et al., 2012; Ratnasingham \& Hebert, 2013). Each specimen with a sequence longer than $500 \mathrm{bp}$ automatically gains a BIN (Barcode Index Number) assignment on BOLD that is based on the RESL algorithm (Ratnasingham \& Hebert, 2013). BINs may be merged when genetically intermediate specimens are added, or split when new records reveal a clear sequence divergence structure. Distance-based neighbour-joining (NJ) was used to reconstruct DNA barcode gene trees. Despite certain limitations, $\mathrm{NJ}$ has repeatedly been shown to perform well for species identification (Huelsenbeck \& Hillis, 1993; Kumar \& Gadagkar, 2000; Mihaescu et al., 2009; Mutanen et al., 2016).

\section{Phylogenetic relationships}

A reconstruction of the phylogenetic relationships of species of Lymanopoda was done using the Maximun Likelihood (ML) method. Two species of Satyrinae were used as an outgroup: Corades chelonis and Lasiophila zapatoza. The analysis was done using the Phylogeny.fr platform (Dereeper et al., 2008, 2010) and sequences were aligned using MUSCLE (v3.8.31) and configured for highest accuracy (MUSCLE with default settings).

The phylogenetic tree was constructed using the ML method implemented in the PhyML program (v3.1/3.0 aLRT). The HKY85 substitution model was selected assuming an estimated proportion of invariant sites (of 0.601) and four gamma-distributed rate categories to account for the percentage heterogeneity across sites. The gamma shape parameter was estimated directly from the data $($ gamma $=1.147)$. The reliability of internal branches was assessed using the aLRT test (SH-Like). The graphical representation and editing of the phylogenetic tree were done using TreeDyn (v198.3).

\section{RESULTS}

\section{Taxonomy}

\section{Genus Lymanopoda Westwood, 1851}

Lymanopoda Westwood, 1851 (May): Pl. LXVII, Figs 6, 7. Type species: L. samius Westwood, 1851, by monotypy.

Sarromia Westwood, 1851 (May): Pl. LXVII, Fig. 5. Type species: S. obsoleta Westwood, 1851, by monotypy. Synonymized by Westwood, 1851 (July): 401-402.

Zabirnia Hewitson, 1877: 92. Type species: Z. zigomala Hewitson, 1877, by monotypy. Synonymized by Pyrcz, 2004: 463. 


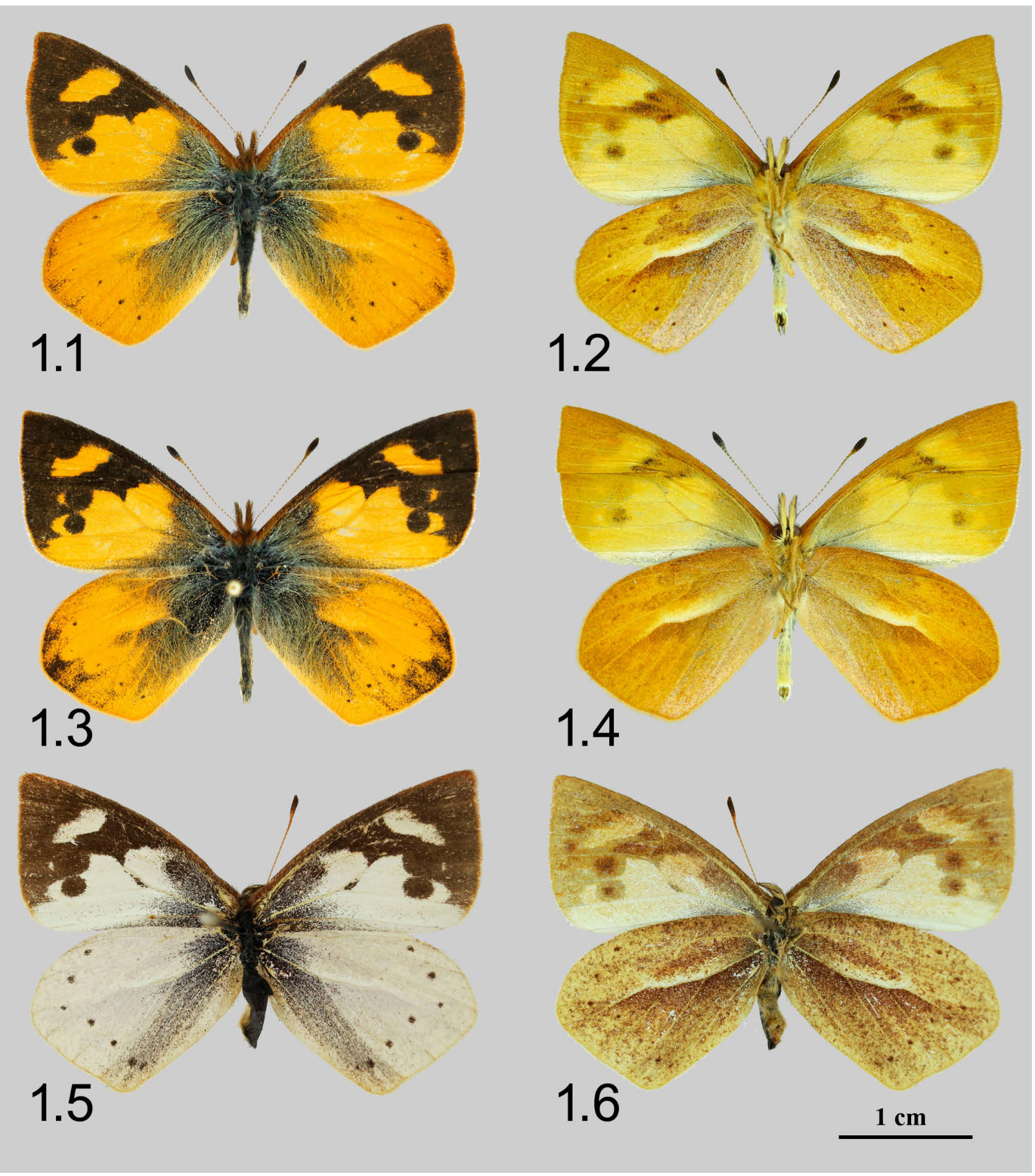

Fig. 1. Adults of Lymanopoda flammigera sp. n. 1.1 - male paratype (upperside); 1.2 - male paratype (underside); 1.3 - male paratype (upperside); 1.4 - male paratype (underside); 1.5 - female paratype (upperside); 1.6 - female paratype (underside).

Trophonina Röber, 1889: 222. Type species: Lymanopoda acraei$d a$ Butler, 1868, by monotypy. Synonymized by Pyrcz, 2004 463.

Sabatoga Staudinger, 1897: 143. Type species: S. mirabilis Staudinger, 1897, by monotypy. Synonymized by Adams \& Bernard, 1977: 270.

\section{Lymanopoda flammigera Pyrcz, Prieto \& Boyer, sp. n.}

(Figs 1.1-1.6, 2.1-2.4, 3.6)
ZooBank taxon LSID:

4B29BAB2-E924-4C1B-AFCA-AF131920FF02

\section{Diagnosis}

This species has the size, wing shape, and wing pattern similar to L. huilana Wreymer, 1911 and L. tolima Weymer, 1890 (depicted in Fig. 5), but males differ from both these species and from any other congener by the golden yellow colour of their upper and undersides. The females are 


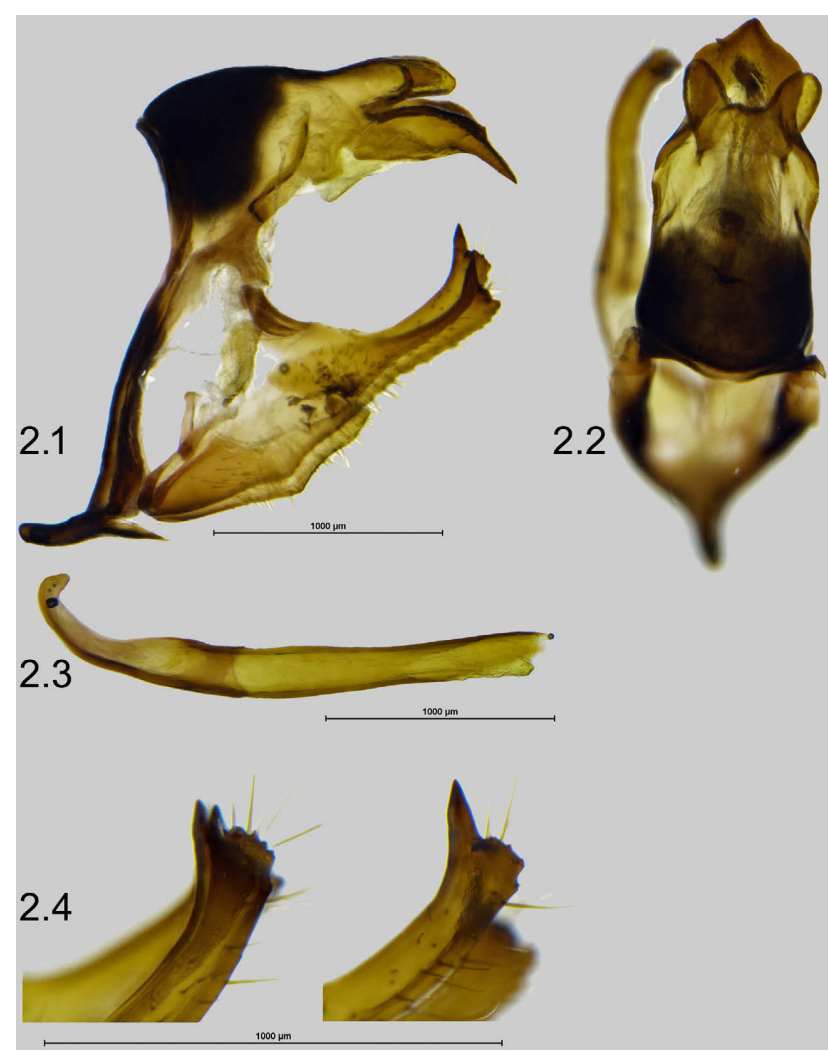

Fig. 2. Male genitalia of Lymanopoda flammigera sp. n. (paratype). 2.1 - lateral view; 2.2 - view from above; 2.3 - aedeagus in lateral view; 2.4 - details of the apices of the right and left valvae in lateral view.

whitish and thus nearly inseparable from the most closely related species, which are, however, not sympatric.

\section{Description}

Male. (Figs 1.1-1.4) Head: Eyes chestnut covered with long, black hairs; labial palps two and a half the length of head, covered with yellow and black hairs, dorsally also brown scales; frons with a tuft of brown hair; antennae reaching half length of the costa, chestnut with white scales at the base of each flagellomere, club composed of 10 segments, strongly flattened and dilated, brown, dorsally slightly lighter with a median groove. Thorax: Dorsally black, mostly naked, with some long but sparse silver hairs, tegulae covered with long, golden brown hairs; ventrally black but covered with long and dense yellow and white hairs; femora of second and third pair of legs black, with first pair and tibiae and tarsi yellow, densely covered with scales. Wings: FW (length: 20-21 mm) triangular with a pointed apex, straight outer margin and shallow tornus; HW oval with a rounded apex and straight outer margin from vein M2 to tornus where bent nearly at a right angle, anal margin straight. FWD yellow of variable shade, between pale yellow (in older individuals) and golden yellow from basal to postmedian area, except for a greyish basal suffusion and an elongated patch in subapical area; distally dark brown with sharp basal notches along the discal cell and vein $\mathrm{Cu} 2 \mathrm{~A}$, a dark brown ocellus in space $\mathrm{Cu} 1 \mathrm{~A}$ $\mathrm{Cu} 2 \mathrm{~A}$. HWD varying between pale yellow and golden yellow with a greyish basal and medial suffusion and with

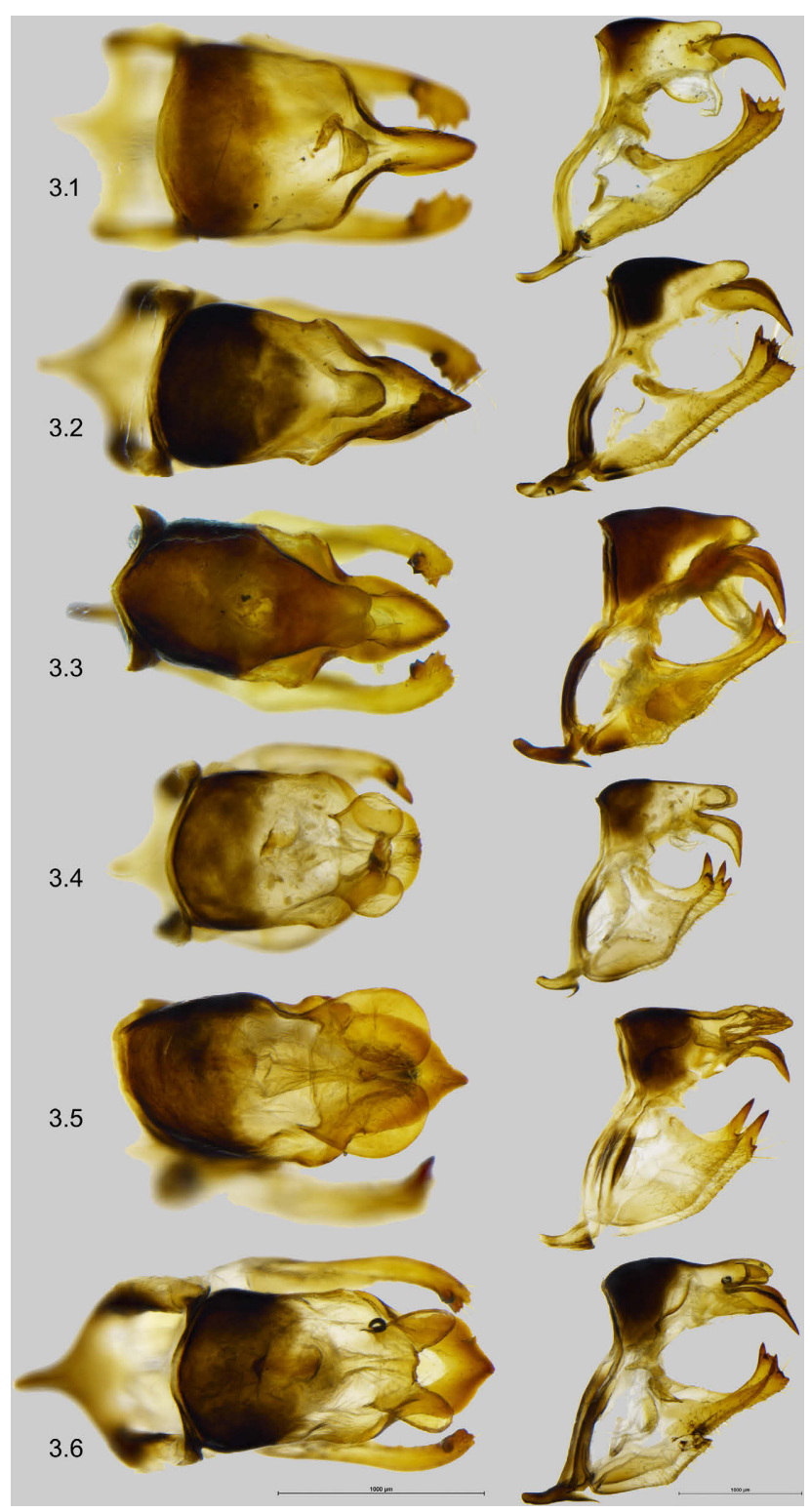

Fig. 3. Male genitalia of "tolima" clade (left: view from above, right: lateral view). 3.1 - Lymanopoda huilana dominicae; $3.2-L$. huilana huilana; $3.3-L$. huilana salazari; $3.4-L$. tolima; $3.5-L$. cassneri; 3.6 - L. flammigera sp. $\mathrm{n}$.

a series of minute, sub marginal black dots (and in some specimens more or less developed marginal dark patches between tornus and apex). FWV colour pattern similar to that on the upper side, but the yellow basal area invariably lighter, and all the dark brown elements are dull and barely visible except for the darker patch in the postdiscal area. HWV light orange almost lacking a pattern except for a lighter, elongated patch in discal cell and a darker brown area immediately behind discal cell; sub marginal tiny black spots as on the upper side. Abdomen: Black dorsally and laterally (covered with dense, velvet black hairs and scales), ventrally with sandy yellow scales and hairs. Genitalia (Figs 2.1-2.4, 3.6): Tegumen strongly sclerotized with a slightly bulged dorsal surface; superuncus prominent, reaching half length of the uncus, bifurcated; uncus stout with a sharp tip pointing downwards; gnathos reduced, blunt; subscaphium small and weakly sclerotized; 
Table 1. Summary of genetic distances among 47 species of Lymanopoda. For each species, the mean and maximum intra-specific values are compared to the nearest neighbour distance. Where the species is represented by a singleton, N/A is displayed for intra-specific values.

\begin{tabular}{|c|c|c|c|c|c|}
\hline Species & $\begin{array}{l}\text { Mean } \\
\text { Intra-Sp }\end{array}$ & $\begin{array}{c}\text { Max } \\
\text { Intra-Sp }\end{array}$ & Nearest species & Nearest neighbour & $\begin{array}{c}\text { Distance } \\
\text { to NN }\end{array}$ \\
\hline Corades chelonis & $\mathrm{N} / \mathrm{A}$ & 0 & Lasiophila zapatoza & GWOTU985-17 & 10.81 \\
\hline Lasiophila zapatoza & N/A & 0 & Corades chelonis & GWOTU974-17 & 10.81 \\
\hline Lymanopoda acraeida & $\mathrm{N} / \mathrm{A}$ & 0 & Lymanopoda venosa & GBGL8234-12 & 5.51 \\
\hline Lymanopoda affineola & $\mathrm{N} / \mathrm{A}$ & 0 & Lymanopoda apulia & GBMIN34627-13 & 2.76 \\
\hline Lymanopoda albocincta & 0.35 & 0.59 & Lymanopoda panacea & GWOTR709-16 & 4.26 \\
\hline Lymanopoda albomaculata & N/A & 0 & Lymanopoda apulia & GBMIN34627-13 & 3.3 \\
\hline Lymanopoda altis & $\mathrm{N} / \mathrm{A}$ & 0 & Lymanopoda confusa & GBMIN34624-13 & 2.59 \\
\hline Lymanopoda apulia & N/A & 0 & Lymanopoda affineola & GBGL8210-12 & 2.76 \\
\hline Lymanopoda araneola & $\mathrm{N} / \mathrm{A}$ & 0 & Lymanopoda shefteli & GBGL8232-12 & 3.59 \\
\hline Lymanopoda caeruleata & $\mathrm{N} / \mathrm{A}$ & 0 & Lymanopoda caucana & GWOTR725-16 & 0.77 \\
\hline Lymanopoda caracara & N/A & 0 & Lymanopoda flammigera & GWOTR859-16 & 6.77 \\
\hline Lymanopoda casneri & $\mathrm{N} / \mathrm{A}$ & 0 & Lymanopoda tolima & GWOTR858-16 & 3.31 \\
\hline Lymanopoda caucana & 0.14 & 0.21 & Lymanopoda caeruleata & GWOTU968-17 & 0.77 \\
\hline Lymanopoda confusa & N/A & 0 & Lymanopoda altis & GWOTR733-16 & 2.59 \\
\hline Lymanopoda dietzi & N/A & 0 & Lymanopoda altis & GWOTR733-16 & 3.3 \\
\hline Lymanopoda eubagioides & $\mathrm{N} / \mathrm{A}$ & 0 & Lymanopoda inde & GBMIN34617-13 & 3.86 \\
\hline Lymanopoda euopis & $\mathrm{N} / \mathrm{A}$ & 0 & Lymanopoda venosa & GBGL8234-12 & 5.51 \\
\hline Lymanopoda excisa & $\mathrm{N} / \mathrm{A}$ & 0 & Lymanopoda pieridina & GWOTR758-16 & 4.39 \\
\hline Lymanopoda ferruginosa & $\mathrm{N} / \mathrm{A}$ & 0 & Lymanopoda shefteli & GBGL8232-12 & 3.3 \\
\hline Lymanopoda flammigera & 0 & 0 & Lymanopoda tolima & GWOTR858-16 & 5.3 \\
\hline Lymanopoda florenciensis & 0.18 & 0.19 & Lymanopoda affineola & GBGL8210-12 & 4.19 \\
\hline Lymanopoda hazelana & N/A & 0 & Lymanopoda samius & GWOTR751-16 & 6.99 \\
\hline Lymanopoda huilana & $\mathrm{N} / \mathrm{A}$ & 0 & Lymanopoda melia & GBGL8226-12 & 4.41 \\
\hline Lymanopoda hyagnis & $\mathrm{N} / \mathrm{A}$ & 0 & Lymanopoda umbratilis & GBGL8233-12 & 0.39 \\
\hline Lymanopoda inde & $\mathrm{N} / \mathrm{A}$ & 0 & Lymanopoda eubagioides & GBMIN34621-13 & 3.86 \\
\hline Lymanopoda ionius & $\mathrm{N} / \mathrm{A}$ & 0 & Lymanopoda pieridina & GWOTR758-16 & 2.94 \\
\hline Lymanopoda labda ssp. & $\mathrm{N} / \mathrm{A}$ & 0 & Lymanopoda araneola & GBGL8213-12 & 4.69 \\
\hline Lymanopoda lecromi & N/A & 0 & Lymanopoda maletera & GWOTR722-16 & 3.06 \\
\hline Lymanopoda magna & N/A & 0 & Lymanopoda obsoleta & GWOTR712-16 & 4.75 \\
\hline Lymanopoda maletera & 0 & 0 & Lymanopoda lecromi & GBGL8224-12 & 3.06 \\
\hline Lymanopoda marianna & $\mathrm{N} / \mathrm{A}$ & 0 & Lymanopoda lecromi & GBGL8224-12 & 7.5 \\
\hline Lymanopoda melia & N/A & 0 & Lymanopoda huilana & GWOTR866-16 & 4.41 \\
\hline Lymanopoda nadia & N/A & 0 & Lymanopoda ferruginosa & GBGL8220-12 & 5.35 \\
\hline Lymanopoda nevada & 0 & 0 & Lymanopoda paramera & GWOTR874-16 & 5.78 \\
\hline Lymanopoda nivea & $\mathrm{N} / \mathrm{A}$ & 0 & Lymanopoda pieridina & GWOTR758-16 & 4.21 \\
\hline Lymanopoda obsoleta & 0.77 & 0.77 & Lymanopoda confusa & GBMIN34624-13 & 2.95 \\
\hline Lymanopoda panacea & N/A & 0 & Lymanopoda apulia & GBMIN34627-13 & 3.31 \\
\hline Lymanopoda paramera & 0 & 0 & Lymanopoda nevada & GWOTR877-16 & 5.78 \\
\hline Lymanopoda pieridina & $\mathrm{N} / \mathrm{A}$ & 0 & Lymanopoda ionius & GWOTR745-16 & 2.94 \\
\hline Lymanopoda prusia & $\mathrm{N} / \mathrm{A}$ & 0 & Lymanopoda tolima & GWOTR858-16 & 9.24 \\
\hline Lymanopoda rana & N/A & 0 & Lymanopoda umbratilis & GBGL8233-12 & 3.94 \\
\hline Lymanopoda samius & 0.1 & 0.15 & Lymanopoda hazelana & GBMIN34619-13 & 6.99 \\
\hline Lymanopoda shefteli & $\mathrm{N} / \mathrm{A}$ & 0 & Lymanopoda hyagnis & GBMIN34618-13 & 1.95 \\
\hline Lymanopoda tolima & 0 & 0 & Lymanopoda casneri & GWOTR869-16 & 3.31 \\
\hline Lymanopoda umbratilis & N/A & 0 & Lymanopoda hyagnis & GBMIN34618-13 & 0.39 \\
\hline Lymanopoda venosa & N/A & 0 & Lymanopoda caucana & GWOTR726-16 & 4.91 \\
\hline Lymanopoda vivienteni & $\mathrm{N} / \mathrm{A}$ & 0 & Lymanopoda ferruginosa & GBGL8220-12 & 7.77 \\
\hline
\end{tabular}

appendix angular, stout but short with a sharp tip; valva elongated, wide in basal half, narrower in the middle, ends with a wide serrated apex and a prominent processus pointing upwards; saccus short and flattened dorso-ventrally; aedeagus simple, tubular, the valva + saccus very slightly arched, with a smooth surface.

Female. (Figs 1.5, 1.6) Sexual dichroism prominent; yellow is replaced by white pigmentation, however the dark brown-blackish elements of the colour pattern are nearly identical, except that they are slightly larger on the FWD, entering more deeply into the discal cell. The HWD sub marginal black dots are also larger. Otherwise, the wing shape of the female differs slightly in being less elongated, especially the hindwings (FW length: $21 \mathrm{~mm}$ ). Female genitalia not examined.

Molecular characterization. No intraspecific haplotype diversity was found in the available sequences $(n=5)$. The lowest overall mean distance to another member of the genus is $5.3 \%$ to L. tolima from Nevado del Ruiz. BIN number: BOLD: ADD7260. Diagnostic fixed states and their position in the COI barcode sequence are depicted in Table 2.

Type material. Holotype male: Colombia, Valle, Tenerife, Páramo Las Domínguez, 3600 m, 29.i.2017, specimen number: i1241, sequence page in BOLD: GWOTU1004-17, C. Prieto. Deposited in ICN. Paratypes, ( $8 \widehat{\varnothing}$ and 19$)$ : 1 : : Colombia, 


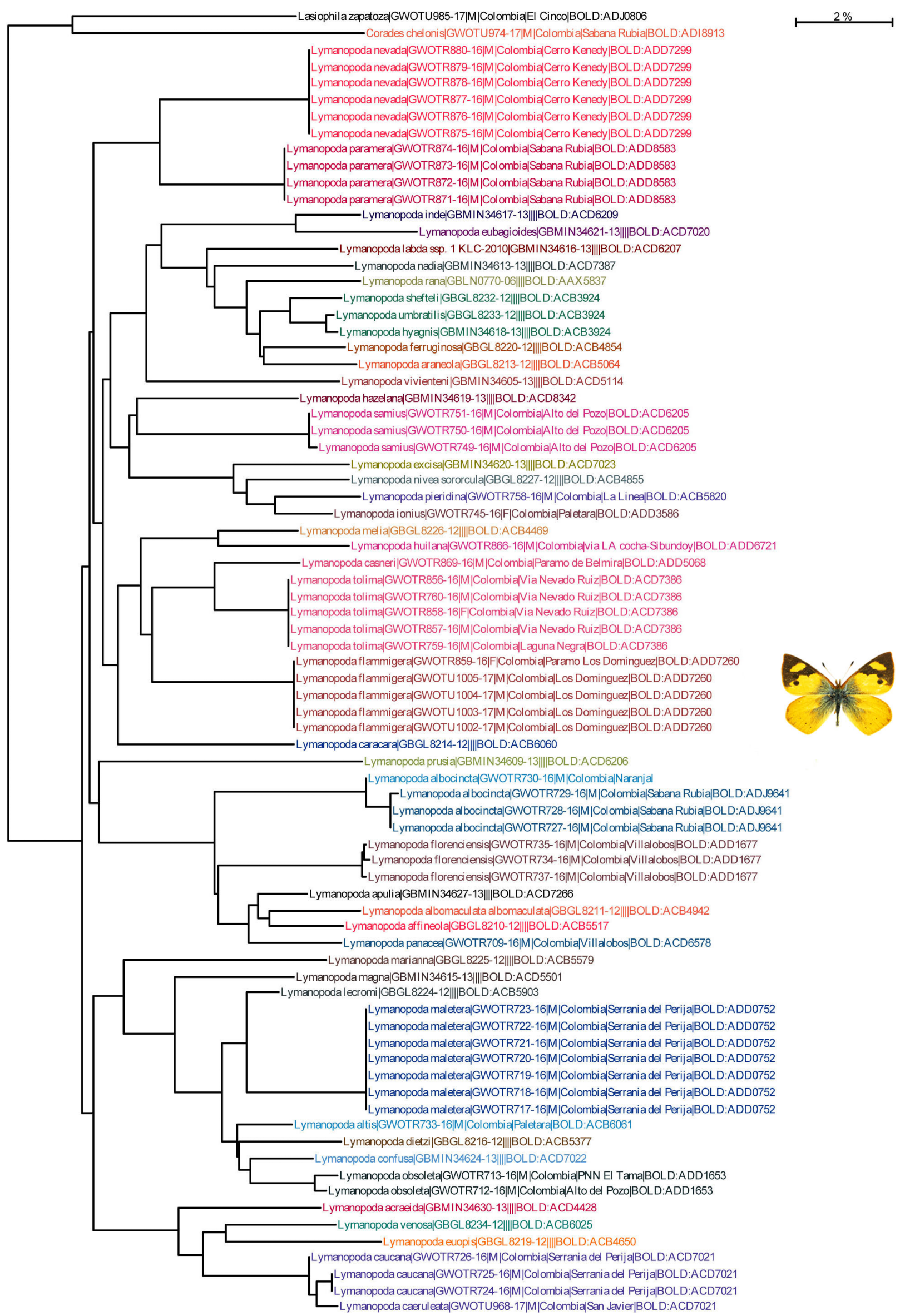

Fig. 4. Neighbour Joining (NJ) identification tree of full-length barcodes (658 bp) for 47 species of Lymanopoda using the K2P-parameter model. BIN (Barcode Index Number) assignment using BOLD is also depicted. 
Table 2. Nucleotide sites with unique fixed states, which serve to identify nine species of Lymanopoda from Colombia. Only species with three or more individuals were included in the analysis. Red - diagnostic characters; orange - partially diagnostic characters.

Nucleotide Position

Group Name

Lymanopoda flammigera

Lymanopoda tolima

Lymanopoda maletera

Lymanopoda samius

Lymanopoda paramera

Lymanopoda nevada

Lymanopoda albocincta

Lymanopoda caucana

Lymanopoda florenciaensis

\section{Group Name}

Lymanopoda flammigera

Lymanopoda tolima

Lymanopoda maletera

Lymanopoda samius

Lymanopoda paramera

Lymanopoda nevada

Lymanopoda albocincta

Lymanopoda caucana

Lymanopoda florenciaensis

\section{Group Name}

Lymanopoda flammigera

Lymanopoda tolima

Lymanopoda maletera

Lymanopoda samius

Lymanopoda paramera

Lymanopoda nevada

Lymanopoda albocincta

Lymanopoda caucana

Lymanopoda florenciaensis

\section{Group Name}

Lymanopoda flammigera

Lymanopoda tolima

Lymanopoda maletera

Lymanopoda samius

Lymanopoda paramera

Lymanopoda nevada

Lymanopoda albocincta

Lymanopoda caucana

Lymanopoda florenciaensis

\section{Group Name}

Lymanopoda flammigera

Lymanopoda tolima

Lymanopoda maletera

Lymanopoda samius

Lymanopoda paramera

Lymanopoda nevada

Lymanopoda albocincta

Lymanopoda caucana

Lymanopoda florenciaensis $\begin{array}{lllllllllllllllllll}43 & 60 & 63 & 66 & 72 & 78 & 84 & 97 & 99 & 114 & 115 & 132 & 139 & 162 & 177 & 201 & 204 & 213 & 216\end{array}$

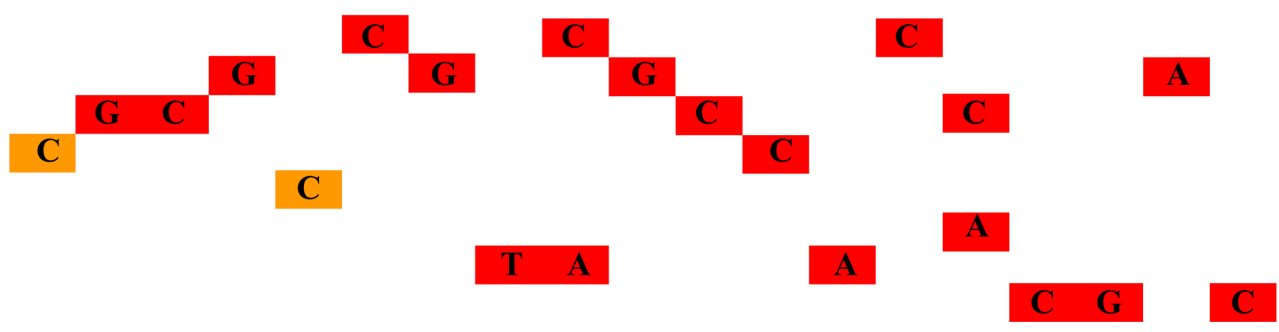

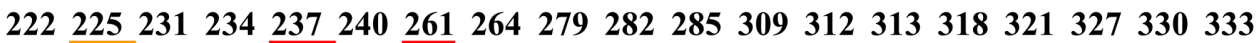
G
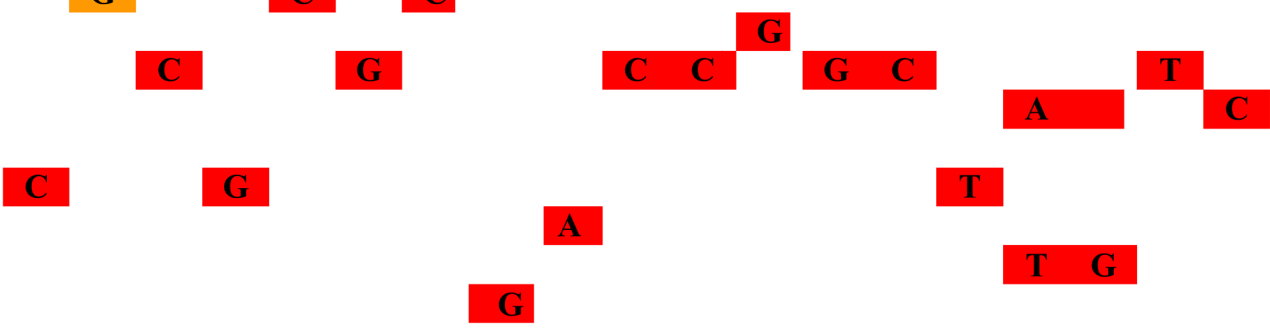

T

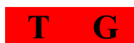

$\begin{array}{lllllllllllllllllll}334 & 349 & 351 & 359 & 366 & 369 & 372 & 381 & 399 & 402 & 405 & 414 & 420 & 429 & 432 & 433 & 439 & 447 & 453\end{array}$ G
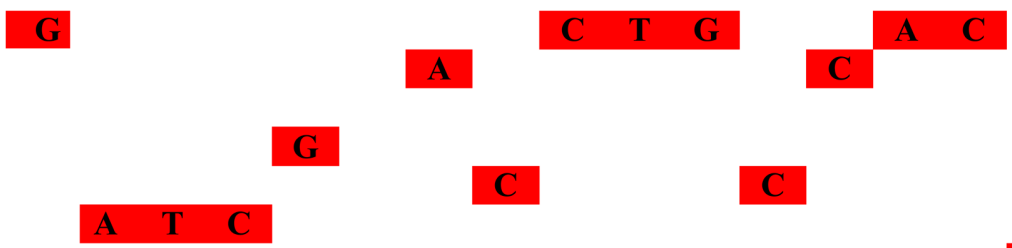

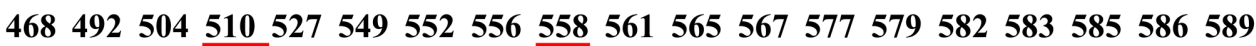

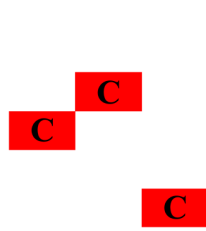
C
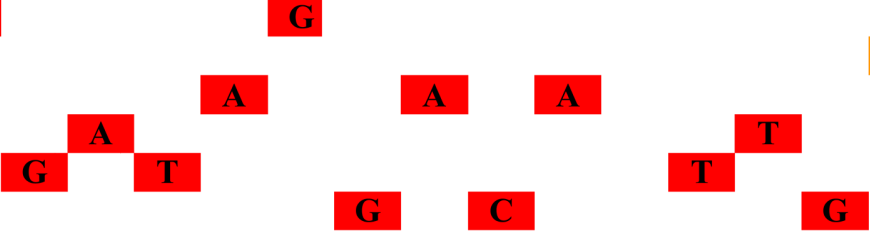

C

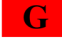

A

C

612615627630639648660669672675678693
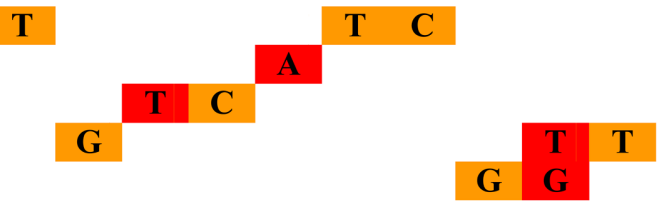

Valle, Tenerife, Páramo Las Domínguez, 3300 m, 21.vii.2013, specimen number: i918, sequence page in BOLD: GWOTR859-

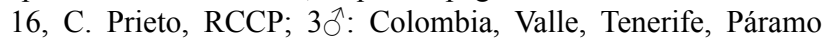
Las Domínguez, 3600 m, 29.i.2017, specimen numbers: i1239, i1240, i1242, sequence pages in BOLD: GWOTU1002-17, GWOTU1003-17, GWOTU1005-17, C. Prieto, RCCP; 30 Colombia, Valle del Cauca, Páramo Las Domínguez, Tenerife, NE Palmira $03^{\circ} 45^{\prime} \mathrm{N}, 7^{\circ} 05^{\prime} \mathrm{W}, 3500-3600 \mathrm{~m}, 29 . \mathrm{i} .2017$, $\mathrm{PB} ; 1 \delta^{\Uparrow}$ 
Table 3. List of specimens, localities and BOLD accession numbers for each individual used in the molecular study.

\begin{tabular}{|c|c|c|c|c|c|c|}
\hline Species & Process ID & Country & Region & Lat & Lon & Elev \\
\hline Lasiophila zapatoza & GWOTU985-17 & Colombia & Perija & 10.3667 & -72.95 & 2200 \\
\hline Corades chelonis & GWOTU974-17 & Colombia & Perija & 10.35 & -72.9167 & 2800 \\
\hline Lymanopoda nevada & GWOTR880-16 & Colombia & Sierra Nevada & 11.1 & -74.0333 & 2800 \\
\hline Lymanopoda nevada & GWOTR879-16 & Colombia & Sierra Nevada & 11.1 & -74.0333 & 2800 \\
\hline Lymanopoda nevada & GWOTR878-16 & Colombia & Sierra Nevada & 11.1 & -74.0333 & 2800 \\
\hline Lymanopoda nevada & GWOTR877-16 & Colombia & Sierra Nevada & 11.1 & -74.0333 & 2800 \\
\hline Lymanopoda nevada & GWOTR876-16 & Colombia & Sierra Nevada & 11.1 & -74.0333 & 2800 \\
\hline Lymanopoda paramera & GWOTR874-16 & Colombia & Serrania del Perija & 10.35 & -72.9167 & 2800 \\
\hline Lymanopoda paramera & GWOTR873-16 & Colombia & Serrania del Perija & 10.35 & -72.9167 & 2800 \\
\hline Lymanopoda paramera & GWOTR872-16 & Colombia & Serrania del Perija & 10.35 & -72.9167 & 2800 \\
\hline Lymanopoda flammigera & GWOTR859-16 & Colombia & Tenerife & 3.68333 & -76.1 & 3600 \\
\hline Lymanopoda tolima & GWOTR858-16 & Colombia & PNN Los Nevados & 4.98333 & -75.3333 & 3200 \\
\hline Lymanopoda tolima & GWOTR857-16 & Colombia & PNN Los Nevados & 4.98333 & -75.3333 & 3200 \\
\hline Lymanopoda tolima & GWOTR856-16 & Colombia & PNN Los Nevados & 4.98333 & -75.3333 & 3200 \\
\hline Lymanopoda tolima & GWOTR760-16 & Colombia & PNN Los Nevados & 4.98333 & -75.3333 & 3200 \\
\hline Lymanopoda flammigera & GWOTU1005-17 & Colombia & Valle, Tenerife & 3.68333 & -76.1 & 3600 \\
\hline Lymanopoda samius & GWOTR751-16 & Colombia & Ocana & 8.03333 & -73.0167 & 2700 \\
\hline Lymanopoda samius & GWOTR750-16 & Colombia & Ocana & 8.03333 & -73.0167 & 2700 \\
\hline Lymanopoda caucana & GWOTR726-16 & Colombia & Manaure & 10.35 & -72.9167 & 1900 \\
\hline Lymanopoda florenciensis & GWOTR735-16 & Colombia & Bota Caucana & 1.51667 & -76.3167 & 1550 \\
\hline Lymanopoda florenciensis & GWOTR734-16 & Colombia & Bota Caucana & 1.51667 & -76.3167 & 1550 \\
\hline Lymanopoda albocincta & GWOTR730-16 & Colombia & Argelia & 2.36667 & -77.1833 & 2700 \\
\hline Lymanopoda albocincta & GWOTR729-16 & Colombia & Manaure & 10.35 & -72.9167 & 2500 \\
\hline Lymanopoda albocincta & GWOTR728-16 & Colombia & Manaure & 10.35 & -72.9167 & 3000 \\
\hline Lymanopoda flammigera & GWOTU1004-17 & Colombia & Valle, Tenerife & 3.68333 & -76.1 & 3600 \\
\hline Lymanopoda flammigera & GWOTU1003-17 & Colombia & Valle, Tenerife & 3.68333 & -76.1 & 3600 \\
\hline Lymanopoda caucana & GWOTR725-16 & Colombia & Manaure & 10.35 & -72.9167 & 1800 \\
\hline Lymanopoda maletera & GWOTR723-16 & Colombia & Villanueva & 10.35 & -72.9167 & 2600 \\
\hline Lymanopoda maletera & GWOTR722-16 & Colombia & Villanueva & 10.35 & -72.9167 & 2600 \\
\hline Lymanopoda maletera & GWOTR721-16 & Colombia & Villanueva & 10.35 & -72.9167 & 2600 \\
\hline Lymanopoda maletera & GWOTR720-16 & Colombia & Villanueva & 10.35 & -72.9167 & 2600 \\
\hline Lymanopoda maletera & GWOTR719-16 & Colombia & Villanueva & 10.35 & -72.9167 & 2600 \\
\hline Lymanopoda maletera & GWOTR718-16 & Colombia & Villanueva & 10.35 & -72.9167 & 2600 \\
\hline Lymanopoda obsoleta & GWOTR713-16 & Colombia & Herran & 7.41667 & -72.4333 & 2400 \\
\hline Lymanopoda venosa & GBGL8234-12 & Perú & Puno & & & 1200 \\
\hline Lymanopoda umbratilis & GBGL8233-12 & Perú & Cuzco & & & 1200 \\
\hline Lymanopoda shefteli & GBGL8232-12 & Perú & Cuzco & & & 2500 \\
\hline Lymanopoda rana & GBLN0770-06 & Perú & Pasco & & & 2500 \\
\hline Lymanopoda prusia & GBMIN34609-13 & Perú & Pasco & & & 2800 \\
\hline Lymanopoda nivea & GBGL8227-12 & Ecuador & Napo & & & 2700 \\
\hline Lymanopoda nadia & GBMIN34613-13 & Ecuador & Morona-Santiago & & & 2800 \\
\hline Lymanopoda melia & GBGL8226-12 & Ecuador & Tungurahua & & & 3600 \\
\hline Lymanopoda marianna & GBGL8225-12 & Venezuela & Merida & & & 3100 \\
\hline Lymanopoda magna & GBMIN34615-13 & Perú & Molinopampa & & & 2870 \\
\hline Lymanopoda lecromi & GBGL8224-12 & Venezuela & Tachira & & & 2700 \\
\hline Lymanopoda labda ssp & GBMIN34616-13 & Colombia & Antioquia & & & 2700 \\
\hline Lymanopoda inde & GBMIN34617-13 & Perú & Molinopampa & & & 3200 \\
\hline Lymanopoda hyagnis & GBMIN34618-13 & Perú & Cuzco & & & 2900 \\
\hline Lymanopoda hazelana & GBMIN34619-13 & Ecuador & Loja & & & 3000 \\
\hline Lymanopoda ferruginosa & GBGL8220-12 & Perú & Cuzco & & & 2050 \\
\hline Lymanopoda excisa & GBMIN34620-13 & Ecuador & Loja & & & 3025 \\
\hline Lymanopoda euopis & GBGL8219-12 & Costa Rica & Irazú & & & 2700 \\
\hline Lymanopoda eubagioides & GBMIN34621-13 & Perú & Cuzco & & & 2600 \\
\hline Lymanopoda dietzi & GBGL8216-12 & Venezuela & Tachira & & & 2700 \\
\hline Lymanopoda confusa & GBMIN34624-13 & Ecuador & Zamora-Chinchipe & & & 2100 \\
\hline Lymanopoda araneola & GBGL8213-12 & Perú & Molinopampa & & & 2870 \\
\hline Lymanopoda apulia & GBMIN34627-13 & Perú & Pasco & & & 2600 \\
\hline Lymanopoda albomaculata & GBGL8211-12 & Bolivia & Cochabamba & & & 2750 \\
\hline Lymanopoda affineola & GBGL8210-12 & Perú & Puno & & & 2700 \\
\hline Lymanopoda acraeida & GBMIN34630-13 & Perú & Cuzco & & & 1400 \\
\hline Lymanopoda vivienteni & GBMIN34605-13 & Colombia & Guasca & & & 3200 \\
\hline Lymanopoda nevada & GWOTR875-16 & Colombia & Sierra Nevada & 11.1 & -74.0333 & 2800 \\
\hline Lymanopoda paramera & GWOTR871-16 & Colombia & Serrania del Perija & 10.35 & -72.9167 & 2800 \\
\hline Lymanopoda casneri & GWOTR869-16 & Colombia & Belmira & 6.65 & -75.6667 & 3100 \\
\hline Lymanopoda huilana & GWOTR866-16 & Colombia & Sibundoy & 1.13333 & -77.0833 & 3000 \\
\hline Lymanopoda tolima & GWOTR759-16 & Colombia & PNN Los Nevados & 4.98333 & -75.3333 & 3200 \\
\hline Lymanopoda pieridina & GWOTR758-16 & Colombia & Calarca & 4.46667 & -75.55 & 3200 \\
\hline Lymanopoda samius & GWOTR749-16 & Colombia & Ocana & 8.03333 & -73.0167 & 2700 \\
\hline Lymanopoda ionius & GWOTR745-16 & Colombia & Purace & 2.16667 & -76.3833 & 3000 \\
\hline Lymanopoda florenciensis & GWOTR737-16 & Colombia & Bota Caucana & 1.51667 & -76.3167 & 1400 \\
\hline Lymanopoda altis & GWOTR733-16 & Colombia & PNN Purace & 2.16667 & -76.3833 & 2900 \\
\hline Lymanopoda albocincta & GWOTR727-16 & Colombia & Manaure & 10.35 & -72.9167 & 3000 \\
\hline Lymanopoda flammigera & GWOTU1002-17 & Colombia & Valle, Tenerife & 3.68333 & -76.1 & 3600 \\
\hline Lymanopoda caucana & GWOTR724-16 & Colombia & Manaure & 10.35 & -72.9167 & 1900 \\
\hline Lymanopoda maletera & GWOTR717-16 & Colombia & Villanueva & 10.35 & -72.9167 & 2600 \\
\hline Lymanopoda obsoleta & GWOTR712-16 & Colombia & Ocana & 8.03333 & -73.0167 & 2700 \\
\hline Lymanopoda panacea & GWOTR709-16 & Colombia & Bota Caucana & 1.51667 & -76.3167 & 1800 \\
\hline Lymanopoda caeruleata & GWOTU968-17 & Colombia & Sierra Nevada & 10.8833 & -74.0167 & 1500 \\
\hline Lymanopoda caracara & GBGL8214-12 & Ecuador & Napo & & & 3600 \\
\hline
\end{tabular}




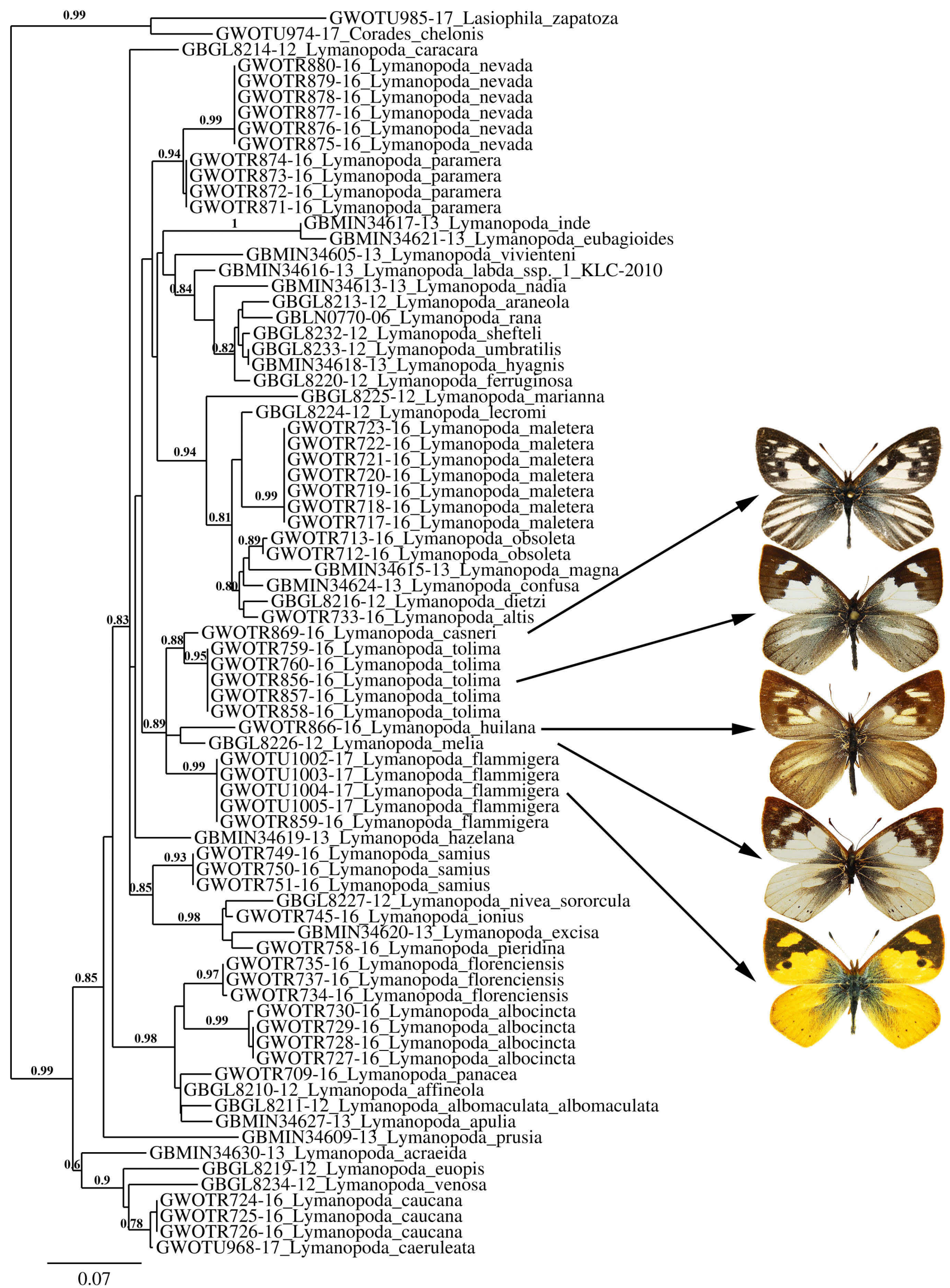

Fig. 5. Phylogenetic tree constructed using the maximum likelihood method implemented in the PhyML program (v3.1/3.0 aLRT) and showing the relationships among taxa belonging to Lymanopoda. Numbers represent branch support values. 
Colombia, Valle del Cauca, Páramo Las Domínguez, Tenerife, NE Palmira $03^{\circ} 45^{\prime} \mathrm{N}, 7^{\circ} 05^{\prime} \mathrm{W}, 3500-3600 \mathrm{~m}, 29 . i .2017$, P Boyer leg., CEP-MZUJ; $1 \delta^{\Uparrow}$ Colombia, Valle del Cauca, Páramo Las Domínguez, Tenerife, NE Palmira $03^{\circ} 45^{\prime} \mathrm{N}, 76^{\circ} 05^{\prime} \mathrm{W}, 3500$ 3600 m, 28.i.2017, P. Boyer leg., prep. genit. 498/14.02.2017 J. Lorenc-Brudecka, CEP-MZUJ.

Type locality. Páramo de Las Dominguez (= Pan de Azúcar), Tenerife, NE Palmira, Valle del Cauca Department, $03^{\circ} 45^{\prime} \mathrm{N}$, $76^{\circ} 05^{\prime} \mathrm{W}, 3500-3600 \mathrm{~m}$ a.s.1.

Etymology. The specific epithet "flammigera" is the nominative feminine singular of "flammiger" from the latin "flamma" (= flame) and -iger (gero) (= to carry, to bear), in reference to the intense orange-yellow colour of the males of this butterfly.

Bionomics. Males patrol at 1-2 $\mathrm{m}$ above the ground in the cloud forest - páramo ecotone. Males patrol around midday along the sunny edges of paths. The immature stages and larval food plants are unknown but are presumed to be Chusquea bamboo, as is the case with other species of Lymanopoda, common in the collecting area.

Distribution. This species is known only from the type locality, Páramo de Las Domínguez (Pan de Azucar in some maps), an isolated massif situated west of the main Colombian Central Cordillera range of the Andes. It occurs in the páramo grassland at 3300-3600 m, just above timberline. Adults were collected in January and July.

\section{Species delimitation based on barcode analysis}

A NJ tree was generated for 47 species and 79 individuals of Lymanopoda. When discrepancies between the DNA-based and standard taxonomy were found, the specimen was examined to confirm that its morphological identification was correct, and the alignment and trace files were carefully re-examined. It was found that 47 morphospecies were assigned to 44 BINs (Fig. 4), therefore showing a $94 \%$ of congruence between morphospecies and BINs. The morphospecies L. hyagnis, L. umbratilis and L. shefteli were placed in the NJ tree under the same BIN code due to the low genetic divergence of $0.39 \%$ between L. hyagnis and L. umbratilis, and $1.9 \%$ between $L$. shefteli and L. hyagnis. Similarly, the genetic divergence between the the morphologically very divergent $L$. caeruleata and L. caucana is $0.77 \%$ (Table 1 ). However, in all the cases, the identification of an unknown specimen by matching its sequence to those in the reference library led to correct results. Nucleotide sites with unique fixed states that were used to identify nine species (those represented by at least three specimens in our dataset) of Lymanopoda from Colombia are compared in Table 2.

The species of Lymanopoda examined have a mean intraspecific genetic distance of $0.05 \%(\mathrm{n}=78$ comparisons of barcodes $>600 \mathrm{bp}$ ). Maximum intraspecific divergence was $0.77 \%$. The mean interspecific genetic distance was $9.60 \%(n=2848$ comparisons of barcodes $>600 \mathrm{bp})$. Maximum interspecific divergence was $13.37 \%$ and minimum interspecific distance was $0.39 \%$.

\section{Lymanopoda phylogeny}

A phylogenetic tree was constructed using the ML method for 47 species of Lymanopoda, including 17 from Colombia and 30 others whose COI sequences were available in GenBank (Fig. 5), out of $\sim 65$ known, which makes up $70 \%$ of all known species. The tree presents four main clades, one of which is called here for convenience "obsoleta" with 14 species including the two species, L. florenciaensis Salazar, Henao \& Vargas, 2004 and L. maletera Adams \& Bernard, 1979, not sequenced before, the "ionius" clade with 17 species, the "caucana" clade with five species and "tolima" clade with eight species. The latter is subdivided into two clades, one of which includes two species not sequenced before, L. nevada Krüger, 1924 and L. paramera Adams \& Bernard, 1979, whereas the other contains six species including L. flammigera sp. n. and two other species not included in the generic phylogeny produced previously (Casner \& Pyrcz, 2010), L. tolima and L. casneri Pyrcz \& Clavijo, 2016, the latter, however, sequenced by Marín et al. (2017). The resolution of this clade is low and presents a polytomy, therefore the position of the new species relative to other five species is not established.

\section{DISCUSSION}

\section{Colour patterns}

The new species is remarkable first of all because of its unusual golden-yellow colour of males, unique not only among other congeners but also within the entire speciesrich subtribe Pronophilina (over 650 species), and arguably among all neotropical and even worldwide Satyrinae. The evolutionary basis of this outstanding colouration is unknown but the hypothesis that this colouring is somehow related to mimicry, seems unlikely. This is because the Sulphur Colias dimera Doubleday, 1847, which is the potential model, although generally very common in the Colombian páramos and probably obnoxious, has not been detected in the region where L. flammigera sp. n. occurs. Other related Colombian species, such as L. huilana, L. tolima, L. zebra Pyrcz \& Rodríguez, 2007, L. casneri and L. melia Weymer, 1911, are predominantly white or black and white, which is certainly associated with thermoregulation and the limited solar radiation at high altitudes, and the higher absorption of UV. It could eventually also prove to be the case for L. flammigera although the optical qualities of its wing pigments and scales have not been investigated so far. It is worth pointing out that there are several similarly pigmented species of skippers (Hesperiidae) in the high tropical and temperate Andes within the genera Zalomes Bell, Wahydra Steinhauser, Hylephila Billberg, and one yet undescribed species of Racta Evans.

It is however puzzling why such unusual colour patterns evolved in just one isolated area whereas throughout the northern and central Andes most páramo species of $L y$ manopoda are predominantly white. On the other hand, it is true that the genus Lymanopoda is particularly plastic phenotypically and a number of species occurring in cloud forests or the forest-páramo ecotone have colour patterns that are unusual for the subfamily Satyrinae, for example, the blue L. hazelana Brown, 1943, L. samius Westwood, 1851 and L. cinna Westwood, 1889, green patched $L$. marianna Staudinger, 1897 or red L. inaudita Pyrcz, 2010. Some of these colour patterns are almost certainly due to 
mimetic relationships, an issue currently being investigated (Pyrcz, in prep.).

\section{Barcoding}

This study provides an initial assessment of the usefulness of DNA barcoding in Lymanopoda. The NJ tree analysis yielded high percentage of correct identifications in the genus Lymanopoda. In the tree, 94\% of the morphospecies used in this study formed distinct clades and were assigned a Barcode Index Number (BIN) matching perfectly the morphology based identifications. In $6 \%$ of the cases, more than one morphospecies shares a BIN number with other species. These cases include five species in this study: $L$. hyagnis, L. umbratilis, L. shefteli cluster together and have the same BIN number; and L. caeruleata and L. caucana also have the same BIN number. The former three species belong to a complex group of morphologically similar taxa occurring allopatrically in parallel valleys in the Madre de Dios upper basin in southern Peru and northern Bolivia, whose relationships are still not fully understood, and their separate specific status is yet to be confirmed by more thorough taxonomic studies involving their spatial, geographic and altitudinal distribution patterns. L. caeruleata and L. caucana are allopatric species, morphologically easily separable by their predominantly blue (L. caeruleata) and brown (L. caucana) wing colour patterns and genital characters, so their separate specific status is strongly supported. Our results confirm that DNA barcoding is a highly efficient method for identifying species in the subfamily Satyrinae, as pointed out in another recent study on high Andean butterflies (Marín et al., 2017).

\section{Phylogeny}

The cladogram based on the COI marker produced for 47 species has to be considered as complementary relative to previous studies as it takes into consideration only one marker, compared to 40 species and 5 molecular markers (Casner \& Pyrcz, 2010). We, however, chose to use only the COI marker because one of the key issues of this study was to investigate the robustness of barcoding relative to morphological traits in evaluating relationships within the genus Lymanopoda, in particular, between hypothetically closely related taxa. It is interesting, from this perspective, to point out that, regarding the subdivision of the genus into main monophyletic groups and, in particular, the basal position of the "caucana" clade comprising 5 species, the results are highly congruent with previous molecular (Casner \& Pyrcz, 2010) and morphological phylogenetic hypotheses (Pyrcz, 2001). The position of L. prusia, Heimlich, 1973, as a sister to the remaining species of Lymanopoda is, however, not confirmed.

The "tolima clade", with 6 species in Casner \& Pyrcz's paper, is here restricted to 4 species, two of which were not previously examined, L. huilana and L. flammigera sp. n. This well supported clade includes all the high altitude páramo species, examined so far, distributed from northcentral Colombia (Belmira) to Ecuador. Also, all of these species share a number of morphological synapomorphies, which support its monophyly. In this respect, the Peruvian species, L. inde Pyrcz, 2004 and L. eubagioides Butler, 1873 , excluded from this clade, stand apart, and their position within this clade suggested originally by Casner \& Pyrcz (op. cit.) should be reconsidered. Importantly, two white páramo species, L. nevada and L. paramera, found in isolated ranges in northern Colombia, were included in the molecular analysis for the first time. Although they superficially resemble the species in the "tolima" clade by being predominantly white, they were placed in a separate clade, even if they still occur in the larger unit comprising the "tolima" clade and not in the other two large clades, "excisa" and "obsoleta".

By combining molecular and morphological data it is possible to determine the closest relatives of L. flammigera sp. n. within the "tolima" clade. COI based analysis is inconclusive in this respect in showing a polytomy. Comparisons of male genitalia show, however, that L. flammigera, L. casneri and L. tolima share a unique synapomorphy, a bifurcate, dorso-ventrally flattened, prominent rounded superuncus. In L. huilana and L. hazelana the superuncus is considerably smaller and not bifurcated even if two lateral lobes are noticeable. Other characters are less evident, although the valvae of $L$. casneri and L. tolima are more similar, being short with a single prominent apical tooth, whereas the valvae of $L$. flammigera $\mathrm{sp}$. n. are narrower in the middle and much longer, looking in this respect more like those of L. huilana. In L. melia, the sister species of $L$. tolima according to Casner \& Pyrcz's (op. cit.) phylogeny, the superuncus is short and single. These data have important phylogeographical implications. L. tolima diverged in the first place from L. huilana even though there is a continuity of páramo habitats between the areas in Quindío, Tolima and Valle del Cauca in the Central Cordillera with those in Cauca and Nariño further south where L. huilana occurs. On the other hand, there are currently no appropriate páramo habitats over $200 \mathrm{~km}$ between Quindio and the Páramo de Belmira in Antioquia where L. casneri is found. Apparently some more complex underlying paleoecological processes have resulted in the shaping of present day distributions of páramo Lymanopoda species in this part of Colombia.

\section{Final considerations}

This study highlights two important facts. It is confirmed that genitalia, in particular those of males, are extremely valuable not only in alpha-taxonomy but also phylogenetically. Here a comparative analysis refines some data obtained using molecular tools. Of course, not in all taxa are male genitalia as informative, which depends mostly on the number of modifications leading to the evolution of noticeable phenotypical traits even in closely related groups of taxa. In the genus Lymanopoda such traits are appreciable. Secondly, our study confirms the usefulness of the COI marker in species definition as well as in phylogenetic considerations, a role that has been questioned. Here, COI support data on 17 species of Colombian Lymanopoda helped refine the phylogeny of the genus, and is congruent in most aspects with the previously proposed arrangement based on five markers. In other words, COI 
does work at least in the genus Lymanopoda, even if in some other taxa of Lepidoptera this may not necessarily be the case. This study expanded our knowledge on the evolution of the genus Lymanopoda by adding seven more species to its phylogeny. Data for several key species are however still missing, in Colombia in particular for L. mirabilis (Staudinger), a high páramo species with unusual extremely elongated wings and atypical genitalia from the southern part of the Cordillera Oriental, and L. melendeza Adams from the Sierra del Cocuy that has some resemblance in both genitalia and colour patterns to the Venezuelan L. marianna Staudinger, known so far only from the holotype.

ACKNOWLEDGEMENTS. For help and support in many ways we acknowledge A. Hausmann (Bavarian State Collection of Zoology, Munich, Germany) and C. Uribe Ortega and L.C. Gutierrez (Universidad del Atlántico, Barranquilla, Colombia). This research was supported by funds from the Georg Forster Research Fellowship Program of the Alexander-von Humboldt Foundation (Bonn), the Federal Ministry for Education and Research (Germany), the Vice-Rectorate for Research of the Universidad del Atlántico, Colombia under decision number 3247 of 12th June 2015, and by an internal grant of the Institute of Zoology and Biomedical Research of the Jagiellonian University, KZDS006320.

\section{REFERENCES}

AdAms M.J. \& BernARD Q.L. 1977: Pronophiline butterflies (Satyridae) of the Sierra Nevada de Santa Marta, Colombia. Syst. Entomol. 2: 263-281.

ButLer A.G. 1868: Catalogue of the Diurnal Lepidoptera of the Family Satyridae in the Collection of the British Museum. British Museum, London, $211 \mathrm{pp}$.

CAsner K.L. \& Pyrcz T.W. 2010: Patterns and timing of diversification in a tropical montane butterfly genus, Lymanopoda (Nymphalidae, Satyrinae). - Ecography 33: 251-259.

Dereeper A., Guignon V., Blanc G., Audic S., Buffet S., Chevenet F., Dufayard J.F., Guindon S., Lefort V., Lescot M., Claverie J.M. \& Gascuel O. 2008: Phylogeny.fr: robust phylogenetic analysis for the non-specialist. - Nucl. Acids Res. 36(suppl. 2): W465-W469.

Dereeper A., Audic S., Claverie J.M. \& Blanc G. 2010: BLASTEXPLORER helps you building datasets for phylogenetic analysis. - BMC Evol. Biol. 10: 8, 6 pp.

deWaArd J.R., Ivanova N.V., Hajibabaei M. \& Hebert P.D.N. 2008: Assembling DNA barcodes: analytical protocols. In Martin C. (ed.): Methods in Molecular Biology: Environmental Genetics. Humana Press, Totowa, pp. 275-293.

Hebert P.D.N., Penton E.H., Burns J.M., Janzen DH. \& HaLlWACHS W. 2004: Ten species in one: DNA barcoding reveals cryptic species in the neotropical skipper butterfly $A s$ traptes fulgurator. - Proc. Natl. Acad. Sci. USA 101: 1481214817.

Hewitson W.C. 1877: Equatorial Lepidoptera Collected by Mr. Buckley. Part V, pp. 81-96. John Van Voorst, London.

HuelsenBeCK J.P. \& Hillis D.M. 1993: Success of phylogenetic methods in the four-taxon case. - Syst. Biol. 42: 247-264.

IVANOVA N.V., DEWAard J.R. \& HeBert P.D.N. 2006: An inexpensive, automation-friendly protocol for recovering high-quality DNA. - Mol. Ecol. Notes 6: 998-1002.

KLots A.B. 1956: Lepidoptera. In Tuxen S.L. (ed.): Taxonomists' Glossary of Genitalia in Insects. Munksgaard, Copenhagen, pp. $97-110$
KRONFORSt M.R. \& PAPA R. 2015: The functional basis of wing patterning in Heliconius butterflies: The molecules behind mimicry. - Genetics 200: 1-19.

Kumar S. \& GadagKar S.R. 2000: Efficiency of the neighborjoining method in reconstructing deep and shallow evolutionary relationships in large phylogenies. $-J$. Mol. Evol. 51: 544-553.

Mallet J. \& Joron M. 1999: Evolution of diversity in warning color and mimicry: polymorphism, shifting balance and speciation. - Annu. Rev. Ecol. Syst. 30: 201-233.

Marín M.A., Cadavid I.C., Valdés L., Álvarez C.F., Uribe S.I., VILA R. \& Pyrcz T.W. 2017: DNA barcoding of an assembly of montane Andean butterflies (Satyrinae): Geographical scale and identification performance. - Neotrop. Entomol. 46514 523.

Mihaescu R., Levy D. \& Pachter L. 2009: Why neighbor-joining works. - Algorithmica 54: 1-24.

Montero F.A \& OrTIz M.P. 2012: Estados inmaduros e historia natural de algunas especies de la subtribu Pronophilina (Nymphalidae: Satyrinae) presentes en el Paramo del Tablazo - Colombia. II. Lymanopoda schmidti Adams, 1986. — Trop. Lepid. Res. 22: 100-109.

Mutanen M., Kivela S.M., Vos R.A., Doorenweerd C., RatnaSingham S., Hausmann A. \& Godfray H.C. 2016: Specieslevel para- and polyphyly in DNA barcode gene trees: Strong operational bias in European Lepidoptera. - Syst. Biol. 65: $1024-1040$.

Puillandre N., Lambert A., Brouillet S. \& Achaz G. 2012: ABGD, Automatic Barcode Gap Discovery for primary species delimitation. - Mol. Ecol. 21: 1864-1877.

Pyrcz T.W. 1999: The E. Krüger collection of pronophiline butterflies, Part 1: Introduction, genera Altopedaliodes to Lymanopoda. - Lambillionea 99: 221-240.

PyrCZ T. W. 2001: Taxonomic Revision and Zoogeographic Analysis of the Genus Lymanopoda Westwood (Lepidoptera, Nympahlidae, Satyrinae). Unpublished doctoral thesis, Institute of Zoology, Jagiellonian University, 250 pp. [in Polish].

Pyrcz T.W. 2003: Notas taxonomicas y zoogeograficas sobre Lymanopoda huilana con la descripcion de una nueva subespecies del sur-este de Ecuador (Satyrinae, Pronophilini). - Bol. Cient. Mus. Hist. Nat. Caldas 7: 235-243.

Pyrcz T.W. 2004: Pronophiline butterflies of the highlands of Chachapoyas in northern Peru: faunal survey, diversity and distribution patterns (Lepidoptera, Nymphalidae, Satyrinae). - Genus 15: 455-622.

Pyrcz T.W. 2005: A new species of Lymanopoda at the southern generic distribution limit on the Pacific slopes of the Andes. Lambillionea 105: 251-256.

Pyrcz T.W. \& Boyer P. 2011: New taxa of pronophiline butterflies of the genus Lymanopoda Westwood from central Peru (Lepidoptera: Nymphalidae: Satyrinae). — Genus 22: 511-521.

PyrcZ T.W. \& Garlacz R. 2012: The presence-absence situation and its impact on the assemblage structure and interspecific relations of Pronophilina butterflies in the Venezuelan Andes (Lepidoptera: Nymphalidae). — Neotrop. Entomol. 41: 186195.

Pyrcz T.W. \& Rodriguez G. 2006: Description of a new remarkable species of Lymanopoda Westwood and identification of a centre of endemism of cloud forest butterflies in Belmira, northern Central Cordillera, Antioquia, Colombia (Lepidoptera: Nymphalidae: Satyrinae). — Genus 17: 291-297.

Pyrcz T.W. \& WoJtusiaK J. 2002: The vertical distribution of pronophiline butterflies (Nymphalidae, Satyrinae) along an elevational transect in Monte Zerpa (Cordillera de Mérida, 
Venezuela) with remarks on their diversity and parapatric distribution. - Glob. Ecol. Biogeogr. 11: 211-221.

Pyrcz T.W., Willmott K. \& Hall J. 1999: Contributions to the knowledge of Ecuadorian Pronophilini, Part 3, three new species and five new subspecies of Lymanopoda. - Genus 10: 497-522.

Pyrcz T.W., Casner K. \& Wojtusiak J. 2009a: Polytypic species of satyrine butterflies in the subparamos and paramos of the Venezuelan Cordillera de Merida, Part 1: Lymanopoda marianna Staudinger. - Genus 20: 507-532.

Pyrcz T.W., Wojtusiak J. \& Garlacz R. 2009b: Diversity and distribution patterns of Pronophilina butterflies (Lepidoptera: Nymphalidae: Satyrinae) along an altitudinal transect in northwestern Ecuador. — Neotrop. Entomol. 38: 716-726.

Pyrcz T.W., Viloria A.L. \& Boyer P. 2010: The obsoleta clade of the genus Lymanopoda Westwood (Lepidoptera, Nymphalidae: Satyrinae). - Folia Entomol. Hungar. 71: 161-195.

Pyrcz T.W., Clavijo A., Uribe S., Marin M.A., Alvarez C.F. \& ZuBeK A. 2016: Páramo de Belmira as an important centre of endemism in the northern Colombian Andes: New evidence from Pronophilina butterflies (Lepidoptera: Nymphalidae, Satyrinae, Satyrini). — Zootaxa 4179: 77-102.
Ratnasingham S. \& Hebert P.D.N. 2013: A DNA-based registry for all animal species: The Barcode Index Number (BIN) system. - PLOS ONE 8: e66213, 16 pp.

RöBER J.K.M. 1885-1892: II. Theil. Die Familien und Gattungen der Tagfalter Systematisch und Analytisch Bearbeitet von E. Schatz. Nach dem Tode des Verfassers Forgesetzt von J. Röber. In Staudinger O. \& Schatz E. (eds): Exotische Schmetterlinge. G. Löwensohn, Fürth, viii +284 pp., 50 pls.

STAUDINGER O. 1897: Neue südamerikanische Tagfalter. Deutsche Entomol. Z. "Iris" 10: 123-151, pls V, VI.

WeStwood J.O. 1851: The Genera of Diurnal Lepidoptera: Comprising their Generic Characters, a Notice of their Habits and Transformations, and a Catalogue of the Species of each Genus. Longman, Brown, Green \& Longmans, London, 412 pp.

Weymer G. 1911: 4. Familie: Satyridae. In Seitz A. (ed.): DieGross-Schmetterlinge der Erde. 5. A. Kernen, Stuttgart, pp. 173-176.

Weymer G. \& MAassen J.P. 1890: Lepidopteren gesammelt auf einer Reise durch Colombia, Ecuador, Perú, Brasilien, Argentinien und Bolivien in den Jahren 1868-1877 von Alphons Stübel. A. Asher, Berlin, 182 pp.

Received January 11, 2018; revised and accepted June 8, 2018 Published online August 16, 2018 